\title{
Polymer stabilization of the smectic C-alpha* liquid crystal phase-Over tenfold thermal stabilization by confining networks of photo-polymerized reactive mesogens
}

\author{
A. Labeeb, ${ }^{1,2}$ H. F. Gleeson, ${ }^{3}$ and T. Hegmann ${ }^{1, a)}$ \\ ${ }^{1}$ Liquid Crystal Institute, Chemical Physics Interdisciplinary Program, Kent State University, Kent, \\ Ohio 44242, USA \\ ${ }^{2}$ Microwave Physics and Dielectrics, National Research Center, Dokki 12622, Egypt \\ ${ }^{3}$ School of Physics and Astronomy, University of Leeds, Leeds LS2 9JT, United Kingdom
}

(Received 22 September 2015; accepted 27 November 2015; published online 9 December 2015)

The smectic $\mathrm{C}^{*}$-alpha $\left(\mathrm{SmC}_{\alpha}{ }^{*}\right)$ phase is one of the sub-phases of ferroelectric liquid crystals that has drawn much interest due to its electro-optical properties and ultrafast switching. Generally observed above the ferroelectric $\mathrm{SmC}^{*}$ phase in temperature, the $\mathrm{SmC}_{\alpha} *$ commonly shows only very narrow phase temperature range of a few degree Celsius. To broaden the $\mathrm{SmC}_{\alpha}{ }^{*}$ phase, polymer stabilization was investigated for thermal phase stabilization. Two different reactive monomers were tested in three mixtures, and all three broadened the temperature range of the $\mathrm{SmC}_{\alpha}{ }^{*}$ phase from $3{ }^{\circ} \mathrm{C}$ to $39^{\circ} \mathrm{C}$. The current reversal method was used to determine the phase existence versus temperature. Moreover, the texture and network structure was studied by polarized optical microscopy and scanning electron microscopy, with the latter revealing the confinement of the smectic layer structure within the porous polymer network. (C) 2015 AIP Publishing LLC.

[http://dx.doi.org/10.1063/1.4937564]

The tilted, chiral smectic $\mathrm{C}$ phase $\left(\mathrm{SmC}^{*}\right)$ of ferroelectric liquid crystals (FLC) was discovered well before the intermediate, antiferroelectric smectic-C sub-phases. ${ }^{1}$ One of the most intriguing of these sub-phases in the series of antiferroelectric phases (e.g., the four layer $\mathrm{SmC}_{\mathrm{FI} 2} *$ and two layer antiferroelectric $\mathrm{SmC}_{\mathrm{A}}{ }^{*}$ phase) is the smectic C-alpha $\left(\mathrm{SmC}_{\alpha}{ }^{*}\right)$ phase. It has drawn much attention because of its electro-optical properties such as fast response time and low rotational viscosity. ${ }^{2}$ The phase usually exists between the non-tilted smectic A phase ( $\mathrm{SmA}$ ) and the tilted $\mathrm{SmC}^{*}$ phase. The $\mathrm{SmC}_{\alpha}{ }^{*}$ phase provides fast switching (about ten times faster than the ferroelectric $\mathrm{SmC}^{*}$ phase). ${ }^{2}$ An asterisk $\left.{ }^{*}\right)$ denotes the chiral nature of these phases, which is apparent in the helical structure adopted in the bulk of the material in the absence of applied external fields. While the $\mathrm{SmC}_{\alpha} *$ features a helical structure analogous to that of the $\mathrm{SmC}^{*}$ phase, it is nonetheless a distinct mesophase. Mach et al $^{3}{ }^{3}$ reported the final structure of the $\mathrm{SmC}_{\alpha}{ }^{*}$ phase using resonance $\mathrm{x}$-ray diffraction and found an extremely short incommensurate helical pitch of just a few layers (Figure 1). Since the $\mathrm{SmC}_{\alpha}$ * commonly persists only over rather narrow temperature intervals (i.e., a few ${ }^{\circ} \mathrm{C}$ ), broadening of the $\mathrm{SmC}_{\alpha} *$ phase temperature range in a series of mixtures would facilitate a much wider range of experimental studies. Chang et al. ${ }^{4}$ studied the thermal stabilization of the $\mathrm{SmC}_{\alpha}{ }^{*}$ phase via the addition of chiral dopants using a ferroelectric liquid crystal exhibiting a $2{ }^{\circ} \mathrm{C}$-wide $\mathrm{SmC}^{*}{ }_{\alpha}$ phase. The authors reported a dramatic increase in the $\mathrm{SmC}_{\alpha} *$ phase temperature range from $2{ }^{\circ} \mathrm{C}$ to $21^{\circ} \mathrm{C}$ at a concentration of the chiral additive of 8 wt. \%. Moreover, all other SmC* subphases disappeared in favor of the $\mathrm{SmC}_{\alpha}{ }^{*}$ phase. ${ }^{4}$ Further

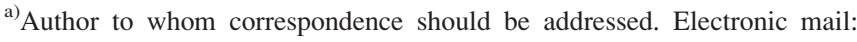
thegmann@kent.edu
}

thermal stabilization of this phase would most certainly contribute to a much better understanding of the $\mathrm{SmC}^{*}$, phase and facilitate the use in commercial device applications. Polymer stabilization of ferroelectric liquid crystals (PSFLC) has recently become an area of great interest in polymer/liquid crystal composites. ${ }^{5}$ These composites are formed by the polymerization of small amounts of reactive monomers (mesogenic or not) that do commonly not exceed $15 \%$ by weight in the liquid crystal host material. ${ }^{6}$ Potential advantages of polymer stabilization in liquid crystals are often enhanced mechanical properties in addition to improved electro-optical characteristics, particularly in nematic and ferroelectric liquid crystals. ${ }^{7}$ Through elastic interactions with smectic host phases, ${ }^{8}$ polymer stabilization can provide bistability, fast switching, and unique mechanical properties as reported by $\mathrm{Walba}^{9}$ and Dierking. ${ }^{10}$ Polymer stabilization has been shown to allow for thermal stabilization of liquid crystal phases as reported by Kikuchi et al. for blue phases. ${ }^{11}$ In these systems, the stabilization arises from the polymer network formation in (after segregation into) the lattice of disclinations, which locks in the blue phase structure over a wide temperature range.

In the current work, polymer stabilization of the $\mathrm{SmC}_{\alpha}$ * phase was tested as a potential avenue to facilitate the broadening of the commonly narrow $\mathrm{SmC}_{\alpha}{ }^{*}$ phase temperature range. Specifically, photo-polymerization of small amounts of reactive monomers dispersed in the host ferroelectric liquid crystal was investigated as described elsewhere. ${ }^{5,11}$ The $\mathrm{SmC}_{\alpha}{ }^{*}$ phase is difficult to differentiate from the $\mathrm{SmC}^{*}$ phase. Although both phases have the same symmetry, these phases have a distinct thermodynamic phase transition between them. ${ }^{12}$ The most reliable and direct indicator to establish the existence of the $\mathrm{SmC}_{\alpha}{ }^{*}$ phase is the current reversal method. ${ }^{13}$ The current reversal response to an applied triangular electric field is two polarization reversal peaks 


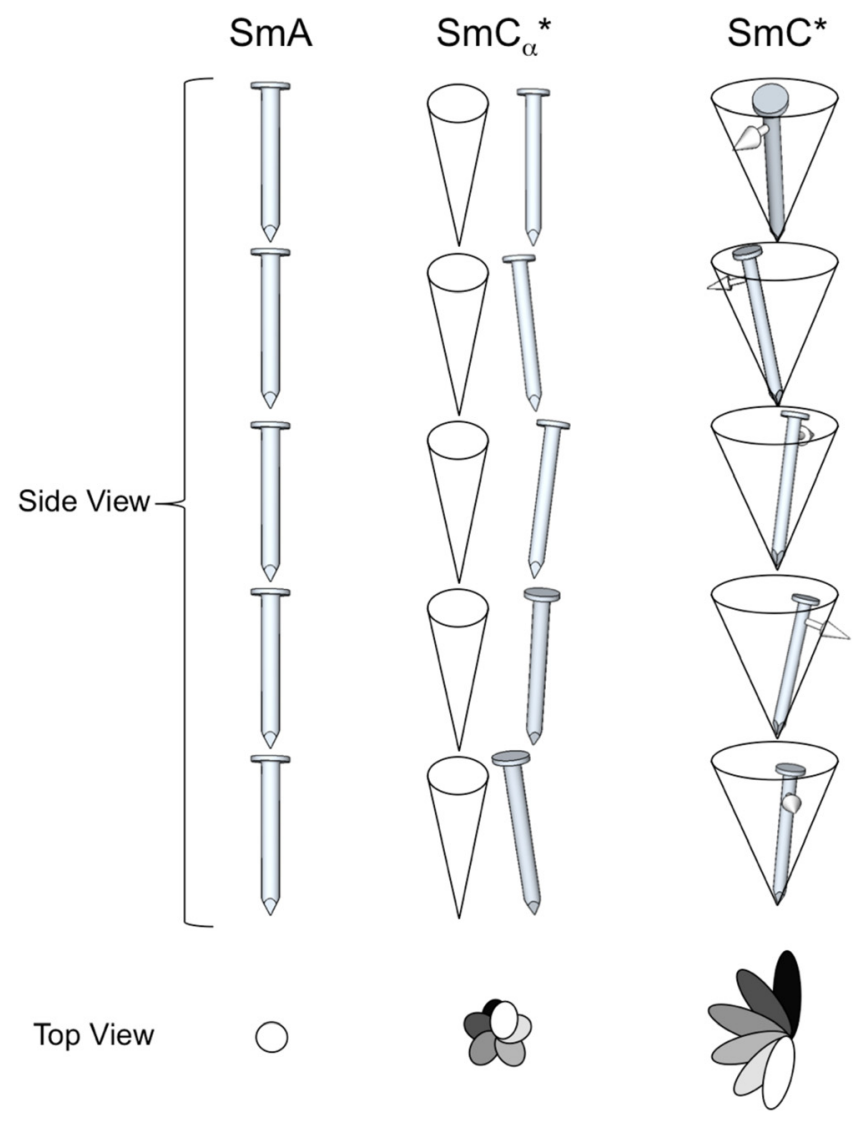

FIG. 1. Illustrations of the director/polarization modulation in the three different smectic phases, the non-tilted $\mathrm{SmA}$, the chiral $\mathrm{SmC}_{\alpha}{ }^{*}$, and the chiral $\mathrm{SmC}^{*}$ phase. In the side-view, a nail represents the director (n) and vector arrows the polarization. In the top view, polarization is omitted and $\mathrm{n}$ is indicated with ellipses (gray shades reflect the layer position). The tilt of the $\mathrm{SmC}_{\alpha}{ }^{*}$ phase is much smaller than in the $\mathrm{SmC}^{*}$ phase.

indicative of an antiferroelectric system. One of the two peaks increases in intensity with decreasing temperature, while the other peak decreases, implying ferrielectric-like character. On further decreasing the temperature, the two asymmetric current peaks merge into a single ferroelectriclike peak indicating the phase transition to the $\mathrm{SmC}^{*}$ phase. Therefore, examining the current response offers a straightforward method to determine the temperature range of the $\mathrm{SmC}_{\alpha} *$ phase.

The FLC material used here is KC983 (Kingston Company, UK) containing 3 wt. \% of a chiral dopant (S1011), as shown in Figure 2. ${ }^{4}$ The reason for choosing this particular FLC system are the widely available data in the literature. ${ }^{4,14-18}$ The chiral dopant increases the $\mathrm{SmC}_{\alpha} *$ temperature range from $1{ }^{\circ} \mathrm{C}$ to $3{ }^{\circ} \mathrm{C}$ while reducing almost all of the phase transition temperatures of the neat $\mathbf{K C 9 8 3}$ material, leading to a phase transition sequence of Iso 94.2 $\mathrm{SmA} 79.1 \mathrm{SmC}_{\alpha} * 76.1$ $\mathrm{SmC}^{*}$ 74.7 $\mathrm{SmC}_{\mathrm{FI} 2}$ * 67.4 $\mathrm{SmC}_{\mathrm{FI} 1}$ * 59.3 $\mathrm{SmC}_{\mathrm{A}} * 33 \mathrm{Cr}$ (on cooling). ${ }^{2,4}$ Two diacrylate monomers were tested: the first monomer was 1,6-hexanediol diacrylate (here termed aliphatic diacrylate, Sigma-Aldrich, USA). The second monomer was 1,4-bis-[4-(3-acryloyloxypropyloxy)benzoyloxy]-2-methylbenzene (here termed reactive mesogen), a nematic liquid crystal monomer (source: Synthon Chemicals $\mathrm{GmbH}$, Germany) with the following phase sequence: Cr 86 N 116 Iso $\left({ }^{\circ} \mathrm{C}\right)$. Both also act as cross-linkers featuring two acrylate

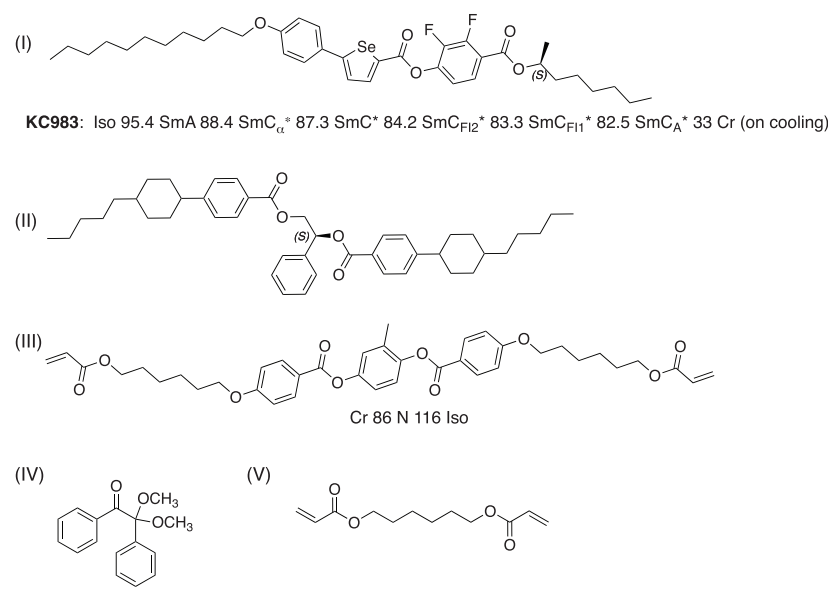

FIG. 2. Chemical structure and thermal phase transition temperature of: (I) host FLC material (KC983), (II) chiral dopant S1011, (III) nematic reactive mesogen, (IV) photo-initiator (Irgacure 651), and (V) aliphatic monomer, 1,6 hexanediol diacrylate.

groups. ${ }^{5}$ As photopolymerization initiator 2,2-dimethyloxy-2phenyl acetophenone, known as Irgacure 651, was used. Three mixture compositions (A, B, and C) were prepared and added to the host FLC at 9 wt. $\%$ aliphatic diacrylate and 1 wt. $\%$ photo-initiator as mixture A (see Table I). Mixture B consisted of 9 wt. $\%$ of aliphatic diacrylate, 1 wt. $\%$ reactive mesogen, and 1 wt. \% photo-initiator. Finally, mixture $\mathrm{C}$ was composed of 9 wt. $\%$ of aliphatic diacrylate, 4 wt. $\%$ reactive mesogen, and $1 \mathrm{wt} . \%$ photo-initiator. The initial mixtures after solvent removal were contained in unidirectional antiparallel rubbed polyimide-coated ITO-glass cells (resistance $100 \Omega$ ) inducing planar alignment with a nominal cell gap of about $5 \pm 0.2 \mu \mathrm{m}$ (Instec, USA). Photopolymerization was initiated using a UV lamp at $365 \mathrm{~nm}(8 \mathrm{~W} / \mathrm{h})$ for at least $30 \mathrm{~min}$. Moreover, to understand how the polymer network stabilizes the $\mathrm{SmC}_{\alpha}{ }^{*}$ phase, scanning electron microscopy (SEM) was employed to reveal the polymer network structure using a Hitachi S-2600N scanning electron microscope. SEM is used after dissolving the liquid crystal host molecules from the polymer network using hexane as a solvent to avoid dissolving the polymer as well. In addition, polarized optical microscopy (POM) reveals the texture of the ferroelectric liquid crystal phase before and after the polymerization using an Olympus BX53 polarizing microscope in conjunction with a Linkam LTS 350 heating/cooling stage.

Prior to photo-polymerization, the addition of the monomers to the host FLC initially causes a large shift in the phase transition temperatures. POM was used to detect

TABLE I. Stabilization of the $\mathrm{SmC}_{\alpha}$ * phase in mixtures differing in composition and monomer concentration.

\begin{tabular}{lccc}
\hline \hline & $\begin{array}{c}\text { Aliphatic } \\
\text { diacrylate } \\
\text { concentration } \\
(\text { wt. \%) }\end{array}$ & $\begin{array}{c}\text { Reactive } \\
\text { mesogen } \\
\text { concentration } \\
\text { (wt. \%) }\end{array}$ & $\begin{array}{c}\text { The } \mathrm{SmC}_{\alpha}{ }^{*} \\
\text { phase temperature } \\
\text { interval }\left({ }^{\circ} \mathrm{C}\right)\end{array}$ \\
\hline Host FLC mix & 0 & 0 & $3 \pm 0.5$ \\
A & 9 & 0 & $6 \pm 0.5$ \\
B & 9 & 1 & $20 \pm 0.5$ \\
C & 9 & 4 & $39 \pm 0.5$ \\
\hline \hline
\end{tabular}



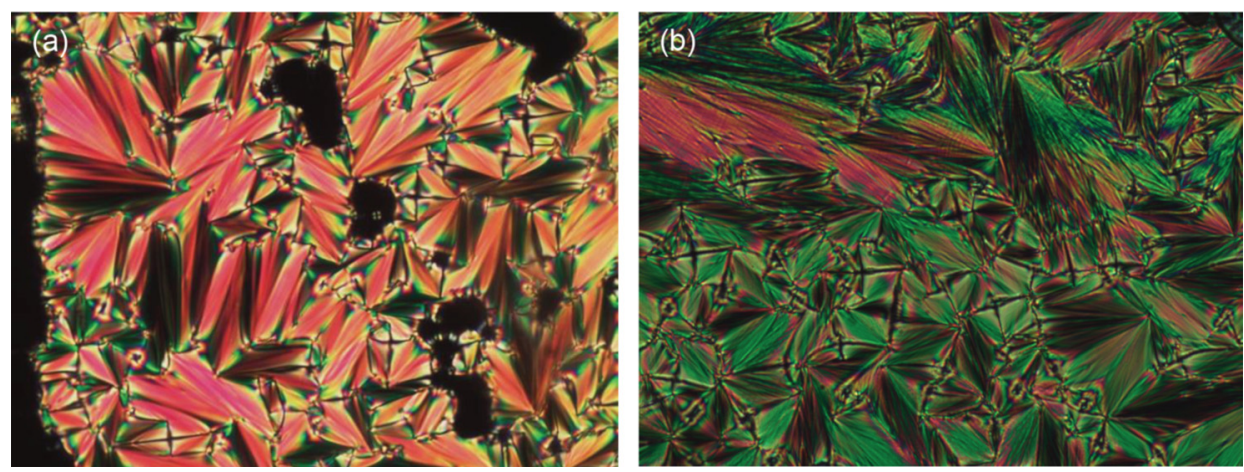

FIG. 3. Representative $\mathrm{SmC}_{\alpha} *$ phase texture under POM (crossed polarizers): (a) before adding monomer and photo-polymerization to host FLC material at $78.6{ }^{\circ} \mathrm{C}$ on cooling and (b) after photo-polymerization at $73.2^{\circ} \mathrm{C}$ on cooling (mixture B).

the onset of the $\mathrm{SmA}-\mathrm{SmC}_{\alpha}{ }^{*}$ phase transition, followed by exposing the cells to UV light at a wavelength of $365 \mathrm{~nm}$ in the $\mathrm{SmC}_{\alpha}$ * phase. Thereafter, the temperature was decreased to accelerate the polymerization rate. ${ }^{5}$ The texture of the $\mathrm{SmC}_{\alpha}{ }^{*}$ phase before polymerization is shown in Figure 3(a). After photopolymerization, the features (domain boundaries) within the texture appear sharper as can be seen in Figure 3 (b), and the phase transition temperatures shifted to lower values. On cooling, the phase transition temperatures for the mixtures after photopolymerization are as follows: Iso 76.7 $\mathrm{SmA} 59.4 \mathrm{SmC}_{\alpha} * 53.2 \mathrm{SmC}^{*} 34 \mathrm{Cr}$. Hence, in the current reversal response at the transition to ferroelectric-like phase only one peak would be expected starting at the transition to the $\mathrm{SmC}^{*}$ phase at $53.2^{\circ} \mathrm{C}$. The current reversal method was then used to determine the phase range of the $\mathrm{SmC}_{\alpha}{ }^{*}$ phase by tracing the appearance of two current reversal peaks. We indeed found that there was a significant increase of the $\mathrm{SmC}_{\alpha}$ * phase temperature range. Mixture B showed the following phase transition temperatures on cooling: Iso 92.4 SmA 80.6 $\mathrm{SmC}_{\alpha}$ * 60.2 SmC* $32 \mathrm{Cr}$. In Figure 4, the current reversal response of mixture $\mathrm{C}$ shows the two characteristic peaks from $72^{\circ} \mathrm{C}$ to $33^{\circ} \mathrm{C}$ on cooling. On further cooling, the mixtures crystallize out, giving the following phase
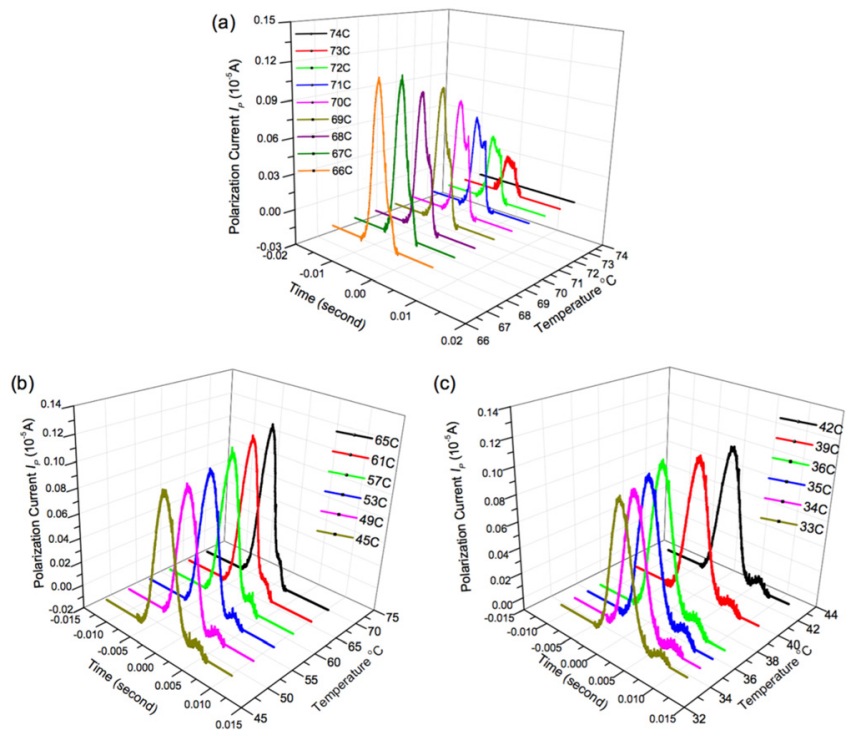

FIG. 4. Current reversal response of mixture $\mathrm{C}$ showing a phase range of $39^{\circ} \mathrm{C}$ of the $\mathrm{SmC}_{\alpha}{ }^{*}$ phase from 72 to $33^{\circ} \mathrm{C}$ on cooling: (a) from $74^{\circ} \mathrm{C}$ to $66^{\circ} \mathrm{C}$, (b) from $65^{\circ} \mathrm{C}$ to $45^{\circ} \mathrm{C}$, and (c) from $42^{\circ} \mathrm{C}$ to $33^{\circ} \mathrm{C}$. The data are manipulated by subtracting both the conducting current due to ions and the current due to resistance of the materials, leaving only the polarization current due to reversibility of electric dipole moments under the applied electric field. sequence on cooling: Iso 92.1 $\mathrm{SmA} 72.3 \mathrm{SmC}_{\alpha} * 33 \mathrm{Cr}$. Thus, the $\mathrm{SmC}_{\alpha} *$ in this particular mixture now exist over a temperature interval of $39^{\circ} \mathrm{C}$-an over tenfold increase from the parent, not polymer-stabilized mixture. The two peaks show the second contribution of the $\mathrm{SmC}_{\alpha} *$ phase, in which the system imitates the ferrielectric-like character as mentioned above. Considering the composition of the three mixtures (A, B, and C), we clearly established that the phase stabilization temperature range increased, especially with an increase in the reactive mesogen concentration. The thermal data for all mixtures collected in Table I illustrate the expansion of the phase temperature range with increasing the reactive mesogen content, with mixture $\mathrm{B}$ showing a $20{ }^{\circ} \mathrm{C} \mathrm{SmC}{ }_{\alpha} *$ temperatures range. Mixture $\mathrm{A}$, in the absence of a photo-polymerized reactive mesogen, shows only a $6^{\circ} \mathrm{C}$ $\mathrm{SmC}_{\alpha} *$ phase range.

One can deduce that the temperature range of the stabilized $\mathrm{SmC}_{\alpha}$ * phase shows a significant increase with increasing concentration of the reactive mesogen. Conversely, the spontaneous polarization $P_{S}$ of the $\mathrm{SmC}_{\alpha}$ * phase, calculated from the reversal current response, decreases dramatically from $45 \pm 2 \mathrm{nC} / \mathrm{cm}^{2}$ for the neat host mixture to around $11 \pm 1 \mathrm{nC} / \mathrm{cm}^{2}$ as shown in Figure 5 and previously reported elsewhere. $^{4,19}$ Mixture B shows a value for the spontaneous polarization of about $16 \pm 1 \mathrm{nC} / \mathrm{cm}^{2}$, which is slightly higher than the $P_{S}$ values found for both mixture A and C. ${ }^{5,20}$ This small increase in $P_{S}$ for mixture B may have its origin in the

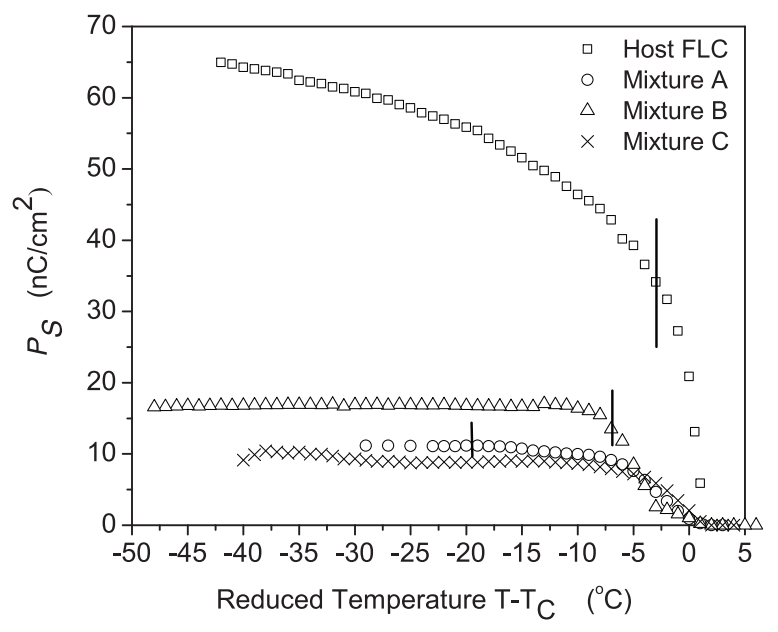

FIG. 5. Plots of the spontaneous polarization $P_{S}$ versus reduced temperature $T-T_{\mathrm{C}}$, where $T$ is measured temperature and $T_{\mathrm{C}}$ is the phase transition temperature from $\mathrm{SmA}^{*}$ to $\mathrm{SmC}^{*}$. The lines refer to the transition from the $\mathrm{SmC}_{\alpha}^{*}$ phase to $\mathrm{SmC}^{*}$ phase, and mixture $\mathrm{C}$ shows only the $\mathrm{SmC}^{*}{ }_{\alpha}$ phase range. 
polymerization conditions with the addition of only a small amount of the reactive mesogen in the presence of a comparatively higher chiral dopant concentration. However, the threefold decrease in $P_{S}$ could best be explained by the incorporation of the acrylate molecules into the smectic layer structure of the FLC host mesogens. The plateau in $P_{S}$ is perhaps best explained by the stabilization of the $\mathrm{SmC}_{\alpha}{ }^{*}$ phase, preventing the FLC mesogens from the formation of and self-assembly into the other subphases, which commonly show higher $P_{S}$ values than the $\mathrm{SmC}_{\alpha}{ }^{*}$ phase. ${ }^{19}$ As soon as the photo-polymerization is initiated by UV light, the polymer network breaks the continuity of the ferroelectric SmC* layer structure and confines the mesogens in the polymer network. $^{21}$ A partial breakdown of the layer structure would then lead to a decoupling of the electric dipoles and perhaps shorten the helical pitch length ${ }^{2}$ and/or increase the elastic constants. ${ }^{4}$ The polymer network consists of porous confinements that prevent the very short incommensurate helical pitch of the $\mathrm{SmC}_{\alpha}{ }^{*}$ phase from its evolution to transit into other $\mathrm{SmC}^{*}$ phase. The relation between the polarization and the helical pitch wave vector $q$ is shown in the critical electric field $E_{c}$, which unwinds the helical pitch length $E_{C}=\pi^{2} K_{3} \theta^{2} q^{2} / 16 P$, where $K_{3}$ is the twist elastic constant, $\theta$ is tilt angle, and $P$ the polarization. ${ }^{22}$ Archer et al. ${ }^{23,24}$ reported the Landau expression showing the relation of the spontaneous polarization and tilt angle to the bilinear and elastic coupling coefficients that increased with increase of the polymer concentration. In turn, this led to a stabilization of the $\mathrm{SmC}_{\alpha}{ }^{*}$ phase and a decrease in $P_{S}$ by increasing the competition between the piezoelectric/flexoelectric effects and interlayer chiral interaction that can unwind the helix without polarization reversal. ${ }^{25}$ Finally, SEM studies provided more obvious clues about the structure of polymeric network and porous confinement structure when the monomer (particularly the reactive mesogen) concentration increased (Figure 6). The polymeric structure confining the liquid crystal host was not observed in mixture A where the reactive mesogen is absent (Figure 6(a)). However, traces of the formation of such confining network can be seen in the SEM images obtained for mixture B (Figure 6(b)). A welldefined network among and across layers was only observed for mixture $\mathrm{C}$ with the highest concentration of the reactive mesogen and the greatest thermal stabilization of the $\mathrm{SmC}_{\alpha}{ }^{*}$ phase, as shown in Figures 6(c) and 6(d). It is worth to note that the stabilized $\mathrm{SmC}_{\alpha}$ * phase (in both mixtures B and C) behaves in a unique way. The phase stabilization does not affect (stabilize) the SmA phase above, in contrast to an earlier report, where an increase in the chiral dopant mole fraction (up to $8 \%$ by weight) stabilized both the SmA and the $\mathrm{SmC}_{\alpha}{ }^{*}$ phase (in fact, at the expense of all other sub-phases, producing a mixture with a $\mathrm{SmC}_{\alpha}{ }^{*}$ to crystalline solid phase transition). ${ }^{4}$ One could imagine that the domain boundaries imposed by the confining polymer network affect either the flexoelectric or piezoelectric coupling (or both) considering the discrete phenomenological model reported by Čepič and co-workers. ${ }^{25,26}$ This would lead to a progressive disappearance of the ferroelectric $\mathrm{SmC}^{*}$ phase leaving only the modulated $\mathrm{SmC}_{\alpha}{ }^{*}$ phase with a short pitch.

In summary, we here report on a previously unobserved, over tenfold thermal polymer stabilization of the $\mathrm{SmC}_{\alpha}$ *
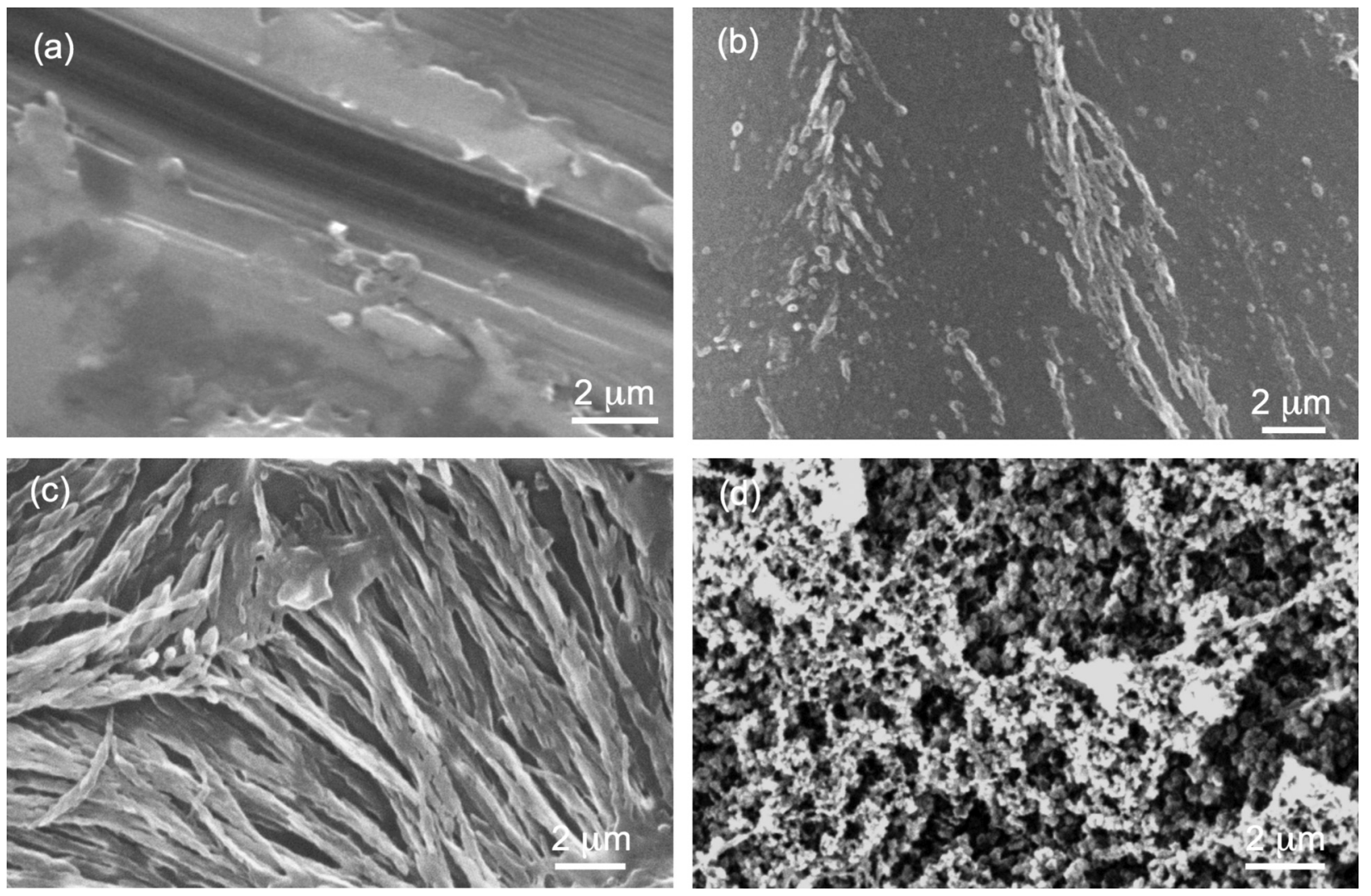

FIG. 6. SEM images of the polymer structure stabilizing the $\mathrm{SmC}_{\alpha}{ }^{*}$ phase for: (a) mixture A, (b) mixture B, and (c) mixture C. The porous, confining polymer structure shown in (d) was obtained for mixture C. 
phase from a $3{ }^{\circ} \mathrm{C}$ to a $39{ }^{\circ} \mathrm{C}$ phase range. The $\mathrm{SmC}_{\alpha} *$ phase stability was determined by the current reversal method. SEM imaging was used to interpret the mechanism of the phase stabilization and revealed that the most stable $\mathrm{SmC}_{\alpha}{ }^{*}$ phase mixtures featured an extensive network among and across layers of the smectic phase. ${ }^{27}$ However, more experiments and tests (including detailed x-ray experiments) of this and related $\mathrm{SmC}_{\alpha} *$ systems are needed to continue improve the temperature range of the $\mathrm{SmC}_{\alpha}{ }^{*}$ phase. Surely, however, the advent of wide temperature range $\mathrm{SmC}_{\alpha} *$ phases with further developments and enhancements could lead to a variety of new applications for and a better understanding of this fast switching phase.

A.L. thanks the Egyptian Education and Cultural Bureau, Egypt Embassy in Washington DC for financial support. T.H. thanks financial support from the Government of Ohio (Ohio Third Frontier Program for Ohio Research Scholars). We also would like to acknowledge the help of A. Jákli for access to instrumentation in his laboratory at the Liquid Crystal Institute, Kent State University, Kent.

${ }^{1}$ A. D. L. Chandani, T. Hagiwara, Y. Suzuki, Y. Ouchi, H. Takezoe, and A. Fukuda, Jpn. J. Appl. Phys., Part 2 27, L729 (1988).

${ }^{2}$ A. Labeeb, PhD thesis, University of Manchester, Manchester, UK, 2011.

${ }^{3}$ P. Mach, R. Pindak, A.-M. Levelut, P. Barois, H. T. Nguyen, H. Baltes, M. Hird, K. Toyne, A. Seed, J. W. Goodby, C. C. Huang, and L. Furenlid, Phys. Rev. E 60, 6793 (1999).

${ }^{4}$ H. S. Chang, S. Jaradat, H. Gleeson, I. Dierking, and M. Osipov, Phys. Rev. E 79, 061706 (2009).

${ }^{5}$ C. A. Guymon, L. Dougan, P. Martens, N. Clark, D. M. Walba, and C. Bowman, Chem. Mater. 10, 2378 (1998).
${ }^{6}$ R. A. Hikmet, H. M. Boots, and M. J. Michielsen, J. Appl. Phys. 79, 8098 (1996).

${ }^{7}$ G. Lester, H. Coles, A. Murayama, and M. Ishikawa, Ferroelectrics 148, 389 (1993).

${ }^{8}$ P. Archer and I. Dierking, J. Opt. A: Pure Appl. Opt. 11, 024022 (2009).

${ }^{9}$ D. M. Walba, Science 270, 250 (1995).

${ }^{10}$ I. Dierking, Materials 7, 3568 (2014).

${ }^{11}$ H. Kikuchi, M. Yokota, Y. Hisakado, H. Yang, and T. Kajiyama, Nat. Mater. 1, 64 (2002).

${ }^{12}$ Z. Q. Liu, S. T. Wang, B. K. McCoy, A. Cady, R. Pindak, W. Caliebe, K. Takekoshi, K. Ema, H. T. Nguyen, and C. C. Huang, Phys. Rev. E 74, 030702(R) (2006).

${ }^{13}$ Y. Takanishi, K. Hiraoka, V. K. Agrawal, H. Takezoe, A. Fukuda, and M. Matsushita, Jpn. J. Appl. Phys., Part 1 30, 2023 (1991).

${ }^{14}$ L. Johnson, S. Jaradat, and H. F. Gleeson, J. Mater. Chem. C 2, 147 (2014).

${ }^{15}$ H. F. Gleeson, S. Jaradat, A. Labeeb, and M. Osipov, Ferroelectrics 431, 40 (2012).

${ }^{16}$ S. Jaradat, P. D. Brimicombe, M. A. Osipov, R. Pindak, and H. F. Gleeson, Appl. Phys. Lett. 98, 043501 (2011).

${ }^{17}$ S. Jaradat, P. D. Brimicombe, S. D. Siemianowski, E. Dimasi, M. A. Osipov, R. Pindak, and H. F. Gleeson, Phys. Rev. E 77, 010701(R) (2008).

${ }^{18}$ S. Jaradat, P. D. Brimicombe, N. W. Roberts, C. Southern, and H. F. Gleeson, Appl. Phys. Lett. 93, 153506 (2008).

${ }^{19}$ S. Jaradat, N. W. Roberts, Y. Wang, L. S. Hirst, and H. F. Gleeson, J. Mater. Chem. 16, 3753 (2006).

${ }^{20}$ S. Kaur, I. Dierking, and H. F. Gleeson, Eur. Phys. J. E 30, 265-274 (2009).

${ }^{21}$ A. D. Gomes, Polymerization (InTech, 2012), p. P17.

${ }^{22}$ A. Levstik, Z. Kutnjak, C. Filipic, I. Levstik, B. Zeks, and T. Carlsson, Phys. Rev. A 42, 2204 (1990).

${ }^{23}$ P. Archer and I. Dierking, J. Phys. D: Appl. Phys. 41, 155422 (2008).

${ }^{24} \mathrm{P}$. Archer, I. Dierking, and M. Osipov, Phys. Rev. E 78, 051703 (2008).

${ }^{25}$ M. Čepič and B. Žekš, Phys. Rev. Lett. 87, 85501 (2001).

${ }^{26}$ N. Vaupotič and M. Čepič, Phys. Rev. E 71, 041701 (2005).

${ }^{27}$ See supplementary material at http://dx.doi.org/10.1063/1.4937564 for Figures S1 to S3 show detailed current reversal data for all mixtures, Figures S4 and S5 show polarized optical microscopy images for all mixtures, and Figures S6 and S7 show SEM images for all three compositions after photopolymerization and washing out the host FLC mixture. 